\title{
PROPORÇÃO DE EXODONTIA E FATORES RELACIONADOS: UM ESTUDO ECOLÓGICO
}

RATE OF TOOTH EXTRACTION AND RELATED FACTORS: AN ECOLOGICAL STUDY

TASA DE EXTRACCIÓN DE DIENTES Y FACTORES RELACIONADOS: UN ESTUDIO ECOLÓGICO

Adriano de Aguiar Filgueira ${ }^{1}$

Angelo Giuseppe Roncalli 2

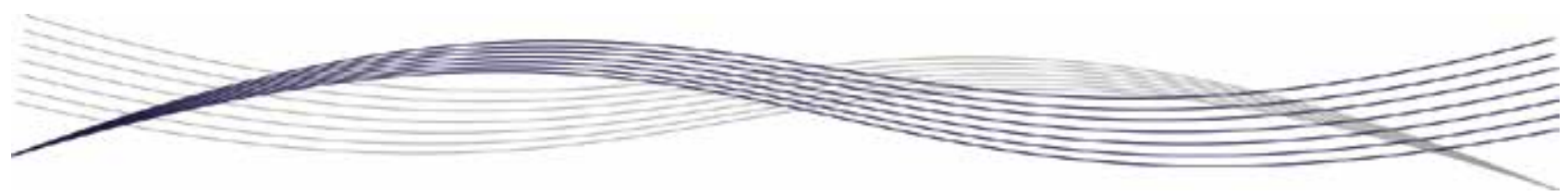

RESUMO

Palavras-chave:

Saúde Bucal; Indicadores

Básicos de Saúde;

Determinantes Sociais de Saúde

Keywords:

Oral Health; Health Status Indicators; Social Determinants of Health.

Palabras clave: Salud Bucal; Indicadores de Salud; Determinantes Sociales de la Salud.

Submetido: 30/08/2018

Aprovado: 30/10/2018

Autor(a) para Correspondência: Adriano de Aguiar Filgueira R. Marechal Deodoro, 55 apto. 205

Benfica - Fortaleza (CE) CEP: 60020-060 E-mail:

adriano.odonto@yahoo.com.br
Condições precárias de saúde bucal, como a perda dentária, representam uma marca de desigualdade social, associando-se a baixa escolaridade $e$ má distribuição de renda. Este artigo analisa a distribuição do indicador relacionado à proporção de exodontia (variável dependente) no Brasil, no periodo de 2011 a 2015, relacionando-o a outros indicadores de saúde e socioeconômicos. Trata-se de estudo ecológico baseado em informações que abrangem todo o território brasileiro, obtidos nos bancos de dados do Departamento de Informática do Sistema Único de Saúde (DataSUS) e do Programa das Nações Unidas para o Desenvolvimento (PNUD). A abordagem estatística foi processada nos programas computacionais SPSS (correlação e regressão linear simples), TerraView (análise espacial pelo Índice de Moran global) e GeoDa (análise espacial bivariada LISA). Os resultados da análise espacial bivariada LISA indicam que a variável dependente se associa a "Média de ação coletiva de escovação dental supervisionada" ( $I=$ -0,300), Indice de Gini $(I=0,481)$ e Índice de desenvolvimento humano (I $=-0,552)$. Elevadas proporções de exodontia foram observadas nas regiões Norte e Nordeste, que apresentaram piores condições socioeconômicas e menos ações de promoção da saúde e prevenção de doenças voltadas à saúde bucal. Concluiu-se que a implantação de políticas públicas para reduzir as iniquidades sociais tende a proporcionar melhores condições de saúde bucal à população.

1. Professor no curso de Odontologia da Universidade Federal do Ceará (UFC). Fortaleza (CE), Brasil. E-mail: adriano.odonto@yahoo.com.br

2. Professor no Departamento de Odontologia e no Programa de Pós-Graduação em Saúde Coletiva da Universidade Federal do Rio Grande do Norte (UFRN). Natal (RN), Brasil. E-mail: roncalli@terra.com.br 


\section{ABSTRACT}

Precarious oral health conditions, such as tooth loss, represent a mark of social inequality, associated with low school education and poor income distribution. This article analyzes the distribution of the indicator related to the rate of tooth extraction (dependent variable) in Brazil, within the period from 2011 to 2015, relating it to other health and socioeconomic indicators. This is an ecological study based on information that covers the entire Brazilian territory, obtained in the databases of the Department of Informatics of the Brazilian National Health System (DataSUS) and the United Nations Development Programme (UNDP). The statistical approach was processed in the softwares SPSS (simple linear correlation and regression), TerraView (spatial analysis by the global Moran index), and GeoDa (LISA bivariate spatial analysis). The results for LISA bivariate spatial analysis indicate that the dependent variable is associated to average collective action of supervised tooth brushing $(I=-0.300)$, Gini index $(I=0.481)$, and Human development index $(I=-0.552)$. High rates of tooth extraction were observed in Northern and Northeastern Brazil, which showed worse socioeconomic conditions and fewer health promotion and disease prevention actions aimed at oral health. It was concluded that the implementation of public policies to reduce social inequities tends to provide the population with better oral health conditions.

\section{RESUMEN}

Condiciones precarias de salud bucal, como la pérdida dental, representan una marca de desigualdad social, asociada con la baja educación escolar y la mala distribución de ingresos. Este artículo analiza la distribución del indicador relacionado con la tasa de extracción de dientes (variable dependiente) en Brasil, en el período de 2011 a 2015, relacionándolo con otros indicadores de salud y socioeconómicos. Este es un estudio ecológico basado en información que cubre todo el territorio brasileño, obtenida en las bases de datos del Departamento de Informática del Sistema Nacional de Salud de Brasil (DataSUS) y del Programa de las Naciones Unidas para el Desarrollo (PNUD). El abordaje estadístico se procesó en los softwares SPSS (correlación y regresión lineal simple), TerraView (análisis espacial mediante el Índice de Moran global) y GeoDa (análisis espacial bivariado LISA). Los resultados del análisis espacial bivariado LISA indican que la variable dependiente está asociada a la acción colectiva promedio del cepillado dental supervisado $(I=-0.300)$, Índice de Gini $(I=0.481)$ e Índice de desarrollo humano $(I=-0.552)$. Se observaron altas tasas de extracción de dientes en el Norte y Nordeste de Brasil, que mostraron peores condiciones socioeconómicas y menos acciones de promoción de la salud y de prevención de enfermedades dirigidas a la salud bucal. Se concluyó que la implementación de políticas públicas para reducir las desigualdades sociales tiende a proporcionar a la población mejores condiciones de salud bucal.

\section{INTRODUÇÃO}

Considerada um problema de saúde pública, a perda dentária é uma das principais consequências das más condições de saúde bucal e representa um efeito cumulativo de doenças bucais. A ausência de dentes leva à diminuição da capacidade mastigatória, gera problemas de fonação e afeta a estética, fato que pode desencadear problemas sociais e psicológicos ${ }^{1}$.

Com o propósito de acompanhar e avaliar as ações e os serviços odontológicos, o primeiro indicador de saúde bucal foi implementado em 1998: “Cobertura de procedimentos preventivos de 0 a 14 anos" 2 . "Proporção de exodontia em relação às ações odontológicas básicas" passou a ser considerado um indicador complementar em 2005, por meio da Portaria GM/MS n. $21^{3}$.

Em 2011, por meio do Decreto n. 7.508, que instituiu o contrato organizativo de ação pública
(COAP) e estabeleceu um rol de diretrizes, objetivos, metas e indicadores, a saúde bucal foi contemplada com três indicadores: "Cobertura populacional estimada pelas equipes básicas de saúde bucal"; "Média da ação coletiva de escovação dental supervisionada"; e "Proporção de exodontia em relação aos procedimentos"4-5. Entretanto, com a Resolução n. 2, de 16 de agosto de 2016, do Ministério da Saúde (MS), apenas o indicador de saúde bucal relacionado às exodontias permaneceu na lista daqueles pactuados para os anos de 2016 a $2019^{6}$.

A exclusão de indicadores de saúde bucal, como o de cobertura populacional, acarreta perdas importantes no que concerne ao planejamento e à avaliação de ações e serviços relacionados à odontologia no setor saúde. 0 indicador de proporção de exodontia indicado no COAP, no entanto, deve ser visto com cautela, pois pode sugerir diversos aspectos, como: 
uma demanda acumulada de extrações dentárias (história natural da cárie dental) devido à falta de acesso aos serviços de odontologia; uma prática mutiladora adotada pelos cirurgiões-dentistas; uma falta de investimento em ações preventivas em saúde bucal de longo prazo; a não priorização por parte dos gestores para a aquisição de materiais e equipamentos que propiciem atividades odontológicas mais conservadoras; além de questões culturais inerentes às diferentes comunidades.

0 conceito de determinantes sociais de saúde (DSS) surgiu nas décadas de 1970 e 80 e ganhou força ao longo dos tempos, sendo amplamente utilizado hoje em diversos estudos para explicar condições de saúde (inclusive de saúde bucal) em diferentes grupos populacionais. Nesse contexto, os problemas de saúde bucal, inclusive a perda dentária, passaram a representar uma marca de desigualdade social, estando associados a baixa escolaridade e má distribuição de renda $a^{7-13}$.

Visando a conhecer a realidade do indicador "Proporção de exodontia em relação aos procedimentos" no território nacional e estabelecer possíveis relações, o objetivo central deste artigo é analisar a distribuição espacial desse indicador de saúde bucal no Brasil no período de 2011 a 2015, relacionando-o a outros indicadores de saúde (“Cobertura populacional estimada pelas equipes de atenção básica"; "Cobertura populacional estimada pelas equipes básicas de saúde bucal"; e "Média de ação coletiva de escovação dental supervisionada") e indicadores socioeconômicos (Índice de Gini e Índice de desenvolvimento humano). Os resultados podem guiar o desenvolvimento de ações e a implementação de políticas públicas para a melhoria das condições de saúde bucais da população de todo o país.

\section{METODOLOGIA}

Trata-se de estudo ecológico realizado com base nas 161 regiões intermediárias de articulação urbana (RIAU) do território brasileiro. 0 Brasil está localizado na América do Sul e tem uma ampla extensão territorial (mais de $8.500 .000 \mathrm{~km}^{2}$ ) e uma população estimada em mais de 206.000.000 de habitantes em 2016. 0 país apresenta fortes disparidades socioeconômicas, sendo as regiões Norte e Nordeste as áreas com piores indicadores socioeconômicos, enquanto as demais regiões apresentam melhores condições de vida ${ }^{14}$.

os dados coletados se referem ao período de

\section{0 Índice de Gini mede o nivel de desigualdade em uma área com base na distribuição de renda...}

2011 a 2015. 0s dados relativos aos indicadores de saúde ("Cobertura populacional estimada pelas equipes de atenção básica"; "Cobertura populacional estimada pelas equipes básicas de saúde bucal"; "Proporção de exodontia em relação aos procedimentos"; e "Média da ação coletiva de escovação dental supervisionada") foram obtidos no site do Departamento de Informática do Sistema Único de Saúde (DataSUS), utilizando os indicadores municipais do Rol de Diretrizes, Objetivos, Metas e Indicadores 2013-2015 do MS ${ }^{15}$.

"Cobertura populacional estimada pelas equipes de atenção básica" é um indicador universal que tem por objetivo medir o acesso aos serviços básicos de saúde, bem como ordenar os cuidados na rede de atenção à saúde, visando à aplicabilidade dos princípios doutrinários do SUS. "Cobertura populacional estimada pelas equipes básicas de saúde bucal" é um indicador universal que visa à ampliação do acesso à saúde bucal pela população. "Proporção de exodontia em relação aos procedimentos" é um indicador específico e mede a qualidade do tratamento ofertado pela odontologia, sendo melhor quanto menor for a proporção de dentes extraídos fato que sugere maior abrangência de procedimentos preventivos e curativos em detrimento de procedimentos mutiladores. Por fim, outro indicador universal utilizado neste estudo foi “Média da ação coletiva de escovação dental supervisionada", que reflete o acesso à orientação para a prevenção de doenças bucais. Os métodos de cálculos dos indicadores apresentados são demonstrados no Caderno de diretrizes, objetivos, metas e indicadores: 2013-2015 do MS ${ }^{16}$.

As informações sociodemográficas (Índice de Gini, Índice de desenvolvimento humano e População) foram obtidas por meio da rede virtual do Programa das Nações Unidas para o Desenvolvimento (PNUD) e correspondem aos dados do Censo Demográfico de $2010^{17}$. 0 Índice de Gini mede o nível de desigualdade em uma área com base na distribuição de renda 
entre sua população, cujos valores variam de 0 (sem desigualdade) a 1 (nível máximo de desigualdade). 0 Índice de desenvolvimento humano é composto e baseia-se em três variáveis (longevidade, educação e renda), com valores que também variam de 0 a 1 quanto mais próximo deste último, maior o Índice de desenvolvimento humano da área.

Todos os dados foram obtidos inicialmente por município brasileiro e, em seguida, foram agrupados no programa computacional SPSS, versão 20, nas respectivas RIAU, para a análise estatística de regressão linear simples. Posteriormente, utilizouse o banco modificado e agregado no programa computacional TerraView, versão 4.2.2, articulado ao arquivo da base cartográfica digital das RIAU, para a análise de dependência espacial pelo Índice de Moran global, o qual estima a autocorrelação espacial (todos os coeficientes nos mapas foram divididos em quintis), além de fornecer o valor $p$ (significância estatística). Utilizou-se o Índice de Moran local para avaliar a presença de clusters, proporcionando a obtenção do BoxMap (independente da significância estatística) e do MoranMap (com significância estatística em $p<0,05)$ da "Proporção de exodontia em relação aos procedimentos".

Com o arquivo produzido no TerraView foi possível realizar a análise bivariada LISA no programa computacional GeoDa, versão 0.9.9.10 (Spatial Analysis Laboratory, University of Illinois, UrbanaChampaign, Estados Unidos da América - EUA). A avaliação da correlação espacial entre a variável de desfecho (“Proporção de exodontia em relação aos procedimentos") e as variáveis independentes (Índice de Gini, Índice de desenvolvimento humano e "Média da ação coletiva de escovação dental supervisionada") foi realizada a partir da construção dos mapas temáticos de cada par de variáveis e da obtenção da significância estatística.

\section{RESULTADOS}

Inicialmente, a partir da amostra composta pelas 161 RIAU existentes no território brasileiro, realizou-se uma análise para verificar a existência de colinearidade entre as variáveis independentes utilizadas no estudo, porém, não foi observada essa relação entre elas.

Em um segundo momento, realizou-se regressão linear simples para avaliar a correlação existente entre a variável dependente e as variáveis independentes (Tabela 1). Essa etapa permitiu identificar uma não correlação significativa entre a proporção de exodontia e os procedimentos e a cobertura populacional estimada pelas equipes básicas de saúde bucal ( $r=0,242 ; p=0,093)$, fato que favoreceu a exclusão da variável "Cobertura populacional estimada pelas equipes básicas de saúde bucal" das demais análises de distribuição e correlação espacial. Apesar da correlação significativa entre os indicadores "Proporção de exodontia em relação aos procedimentos" e "Cobertura populacional estimada pelas equipes básicas de saúde bucal", ela se mostrou muito fraca, o que também acarretou a retirada da segunda variável das análises subsequentes.

Tabela 1 - Análise de regressão linear simples do indicador de Proporção de exodontia em relação aos procedimentos e às variáveis independentes por regiões intermediárias de articulação urbana

\begin{tabular}{lcccc}
\hline \multirow{2}{*}{ Indicadores } & \multicolumn{2}{c}{ Proporção de exodontia em relação aos procedimentos } \\
\cline { 2 - 5 } & $\mathbf{R}$ & IC $95 \%$ & $\mathbf{F}$ & Valor $\mathbf{p}$ \\
\hline $\begin{array}{l}\text { Cobertura populacional estimada pelas } \\
\text { equipes da atenção básica }\end{array}$ & 0,159 & $0,002 / 0,138$ & 4,150 & 0,043 \\
$\begin{array}{l}\text { Média da ação coletiva de escovação } \\
\text { dental supervisionada }\end{array}$ & $-0,352$ & $-1,018 /-0,419$ & 22,439 & $<0,001$ \\
$\begin{array}{l}\text { Índice de Gini } \\
\text { Índice de desenvolvimento humano }\end{array}$ & $-0,552$ & $58,123 / 94,146$ & 69,694 & $<0,001$ \\
\hline
\end{tabular}

Observou-se, no entanto, uma correlação inversamente proporcional, porém de intensidade fraca, entre as variáveis "Proporção de exodontia em relação aos procedimentos" e "Média da ação coletiva de escovação dental supervisionada". Entre as variáveis "Proporção de exodontia em relação aos procedimentos" e Índice de desenvolvimento humano também se observou uma relação de intensidade moderada, indicando que o aumento do Índice de desenvolvimento humano de uma RIAU sugere uma redução da proporção de exodontia em relação aos demais procedimentos odontológicos. Já em relação às variáveis “Proporção de exodontia em relação aos procedimentos" e Índice de Gini se verificou uma correlação diretamente proporcional, sinalizando 
que o aumento da desigualdade da distribuição de renda de uma RIAU pode levar ao aumento da proporção de exodontia.

Em relação à distribuição espacial das variáveis, o indicador de proporção de exodontia em relação aos procedimentos se mostrou mais elevado na maioria das regiões intermediárias de articulação urbana localizadas nas regiões Norte e Nordeste, sendo mais baixo nas RIAU das regiões Centro-0este, Sudeste e Sul, com Índice de Moran global $=0,465$ e estatisticamente significativo $(p=0,01)$ (Figura $1 \mathrm{a})$. A média da ação coletiva de escovação dental supervisionada apresentou um padrão de distribuição espacial fraco $($ Moran $=0,155 ; p=0,03)$ (Figura 1b). No entanto, verificou-se um padrão moderado de distribuição espacial do Índice de Gini (Moran = 0,$660 ; p=0,01$ ) e do Índice de desenvolvimento humano (Moran $=0,779 ; p=0,01$ ), sendo as regiões Norte e Nordeste as que apresentaram maior desigualdade na distribuição de renda e menor Índice de desenvolvimento humano (figuras $1 \mathrm{c}$ e $1 \mathrm{~d}$ ).

1a) Proporção de exodontia em relação aos procedimentos

1b) Média da ação coletiva de escovação dental supervisionada
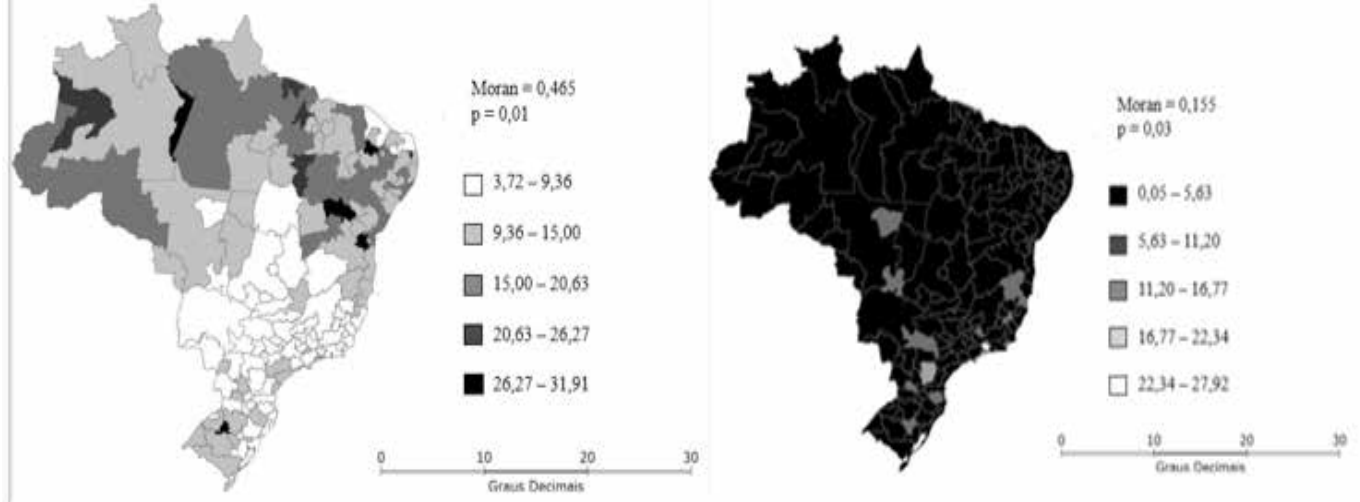

1c) Indice de Gini

Id) Índice de Desenvolvimento Humano
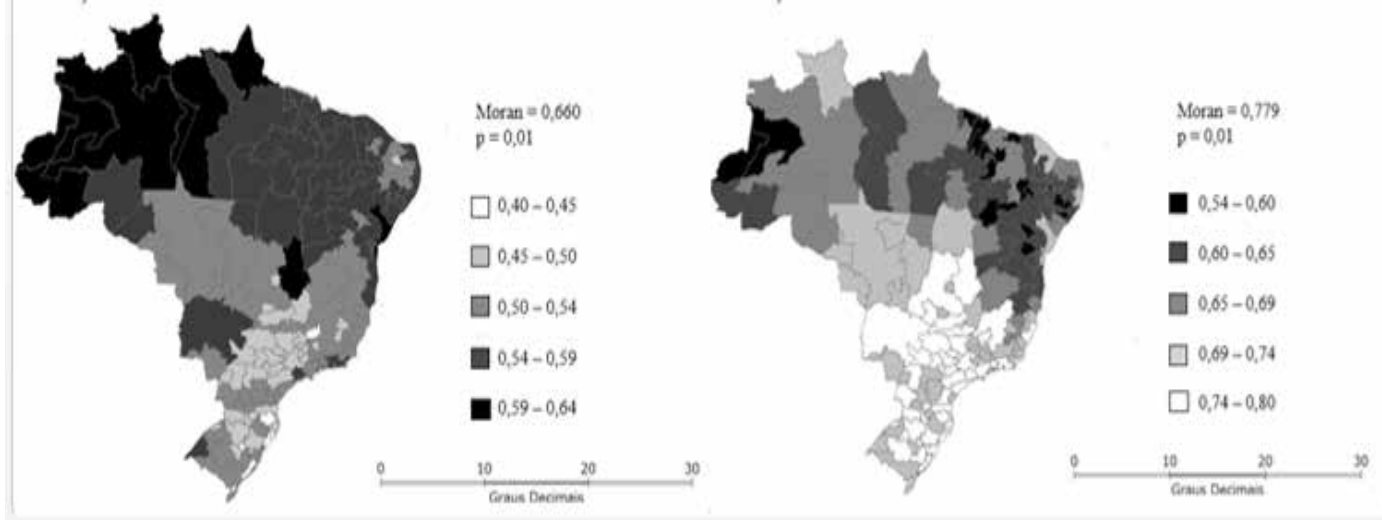

Figura 1. Distribuição espacial de Proporção de exodontia em relação aos procedimentos (1a), Média de ação coletiva de escovação dental supervisionada (1b), Índice de Gini (1c) e Índice de desenvolvimento humano (1d), com os respectivos valores do Índice de Moran global e sua significância estatística. Regiões intermediárias de articulação urbana.

0 BoxMap e o MoranMap da proporção de exodontia relativos aos procedimentos tiveram formação de clusters alto-alto predominantemente no Norte e Nordeste do país (figuras 2 a e 2 b). 


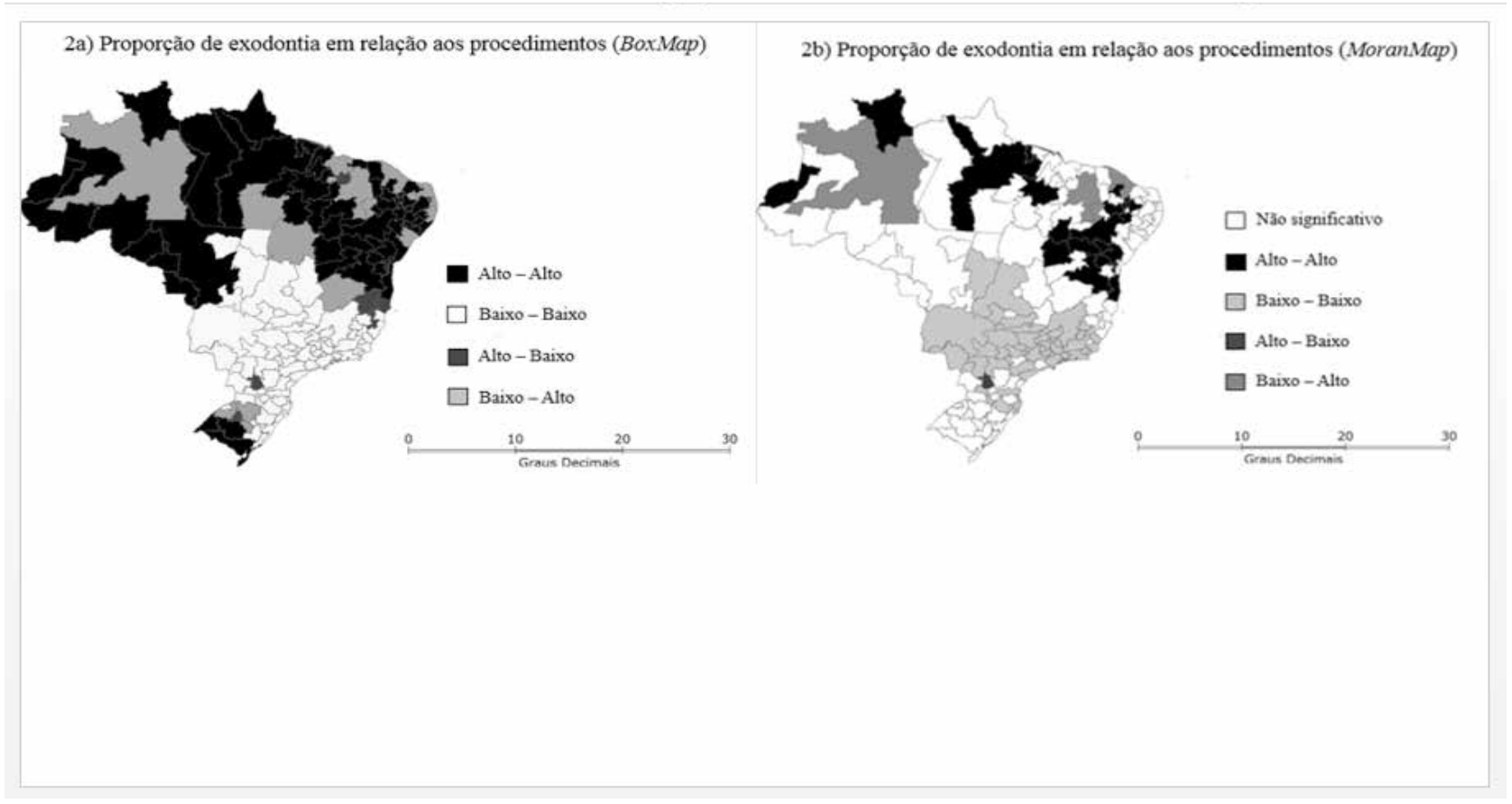

Figura 2. Distribuição espacial dos clusters da Proporção de exodontia em relação aos procedimentos sem (BoxMap) e com LISA estatisticamente significativo (MoranMap). Regiões intermediárias de articulação urbana.

A análise da correlação espacial bivariada obteve as seguintes correlações: fraca negativa (Moran = 0,300 ) entre os indicadores "Proporção de exodontia em relação aos procedimentos" e "Média da ação coletiva de escovação dental supervisionada"; moderada positiva (Moran = 0,481) entre "Proporção de exodontia em relação aos procedimentos" e Índice de Gini; e moderada positiva (Moran = - 0,552) entre "Proporção de exodontia em relação aos procedimentos" e Índice de desenvolvimento humano (figuras 3a, 3b e 3c).
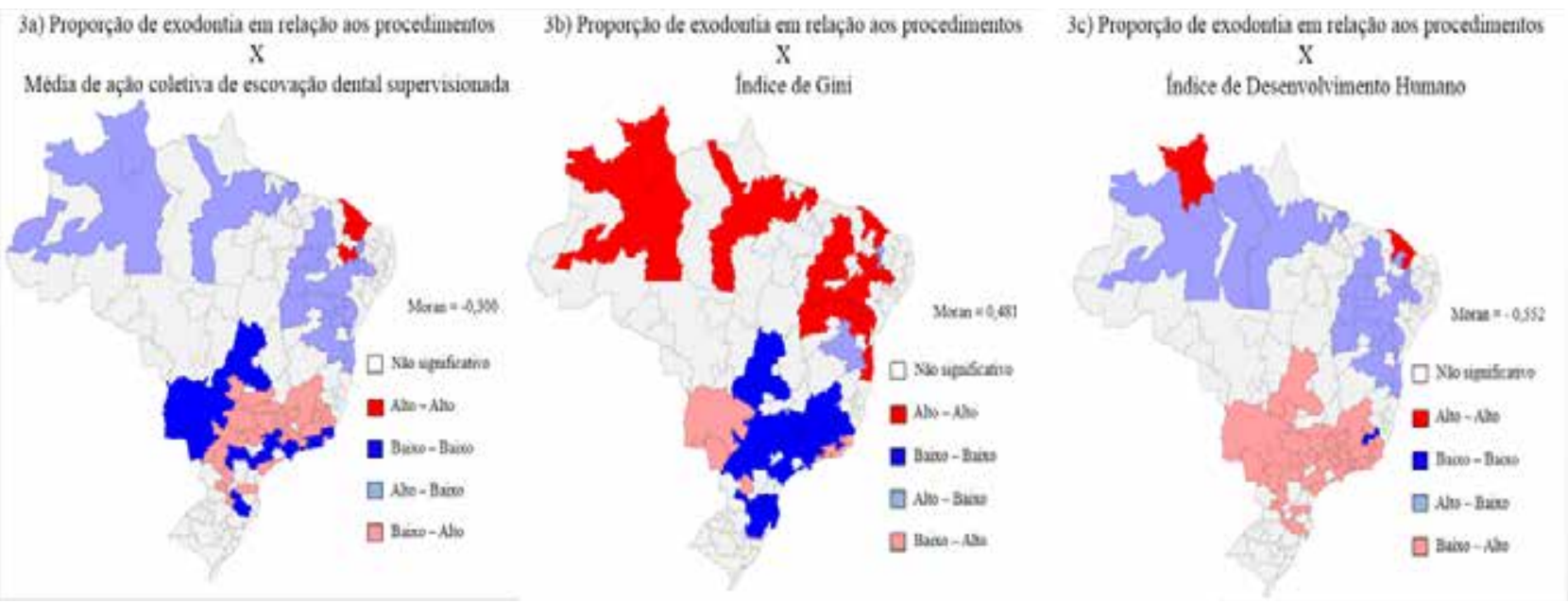

Figura 3. Distribuição dos clusters da correlação espacial bivariada LISA da Proporção de exodontia em relação aos procedimentos com a Média de ação coletiva de escovação dental supervisionada, Índice de Gini e Índice de desenvolvimento humano. Regiões intermediárias de articulação urbana.

Observou-se, portanto, que quanto maior a média da ação coletiva de escovação dental supervisionada e mais elevado o Índice de desenvolvimento humano, menor será a Proporção de extração dentária em relação aos procedimentos preventivos e curativos, sendo a relação deste com o Índice de desenvolvimento humano considerada moderada, enquanto sua relação com a Média de ação coletiva de escovação dental supervisionada é tida como fraca. A correlação entre "Proporção de exodontia em relação aos procedimentos" e Índice de Gini 
também se mostrou moderada (Moran $=0,481$ ), porém, com uma relação que aponta aumento da proporção de exodontia à medida que aumenta a desigualdade na distribuição de renda em determinada população.

\section{DISCUSSÃO}

Conhecida como "Brasil Sorridente", a Política Nacional de Saúde Bucal (PNSB), lançada em 17 de março de 2004, tem como um dos seus objetivos implantar um modelo assistencial com foco nas ações de promoção da saúde e prevenção de doenças em substituição às práticas curativas e mutiladoras ${ }^{18}$. Apesar da melhoria dos indicadores de saúde bucal nos últimos anos, em especial o de proporção de exodontia em relação aos demais procedimentos odontológicos individuais, ainda são perceptíveis fortes desigualdades entre as regiões brasileiras.

A pesquisa nacional, intitulada SB Brasil 2010: Pesquisa Nacional de Saúde Bucal - resultados principais examinou mais de 37.500 brasileiros das mais diversas faixas etárias e espalhados nas 26 capitais estaduais, no Distrito Federal e em 150 municípios do interior com diferentes portes populacionais. 0s resultados apontam fortes diferenças regionais, onde as piores condições de saúde bucal, as médias mais altas de dentes cariados e perdidos, a maior proporção de pessoas que procuram os serviços odontológicos para realizar exodontias e a maioria dos que referem dores dentais mais severas se concentram, principalmente, no Norte e Nordeste do país ${ }^{19}$.

Segundo dados do DataSUS para o ano de 2015, as regiões Norte e Nordeste apresentaram o indicador "Proporção de exodontia em relação aos procedimentos" igual a $19,83 \%$ e $14,40 \%$, respectivamente, bem acima da média nacional $(9,83 \%)$. Além das diferenças culturais entre as regiões do país, é necessário avaliar se a falta de acesso aos serviços odontológico ao longo do tempo gerou demanda acumulada de extrações dentárias, se os cirurgiões-dentistas continuam a adotar uma prática mutiladora e/ou se os gestores não priorizam os investimentos destinados às ações preventivas em saúde bucal e à aquisição de materiais e equipamentos que propiciem ações odontológicas mais conservadoras.

Estudos apontam que, apesar do aumento da proporção de pessoas que conseguem agendar uma consulta odontológica, o acesso/tempo de espera por consultas especializadas ainda representa um grande problema na rede de atenção e que as

\section{As barreiras geográficas também podem ser citadas como fatores dificultadores de acesso aos serviços odontológicos.}

regiões Norte e Nordeste apresentam menor garantia de continuidade do tratamento ${ }^{16-17}$. Esse fato, no entanto, não pode ser justificado pela ausência de serviços especializados, pois, segundo estudo publicado em 2010, o Sudeste, o Centro-0este e o Nordeste apresentavam os maiores percentuais de municípios cobertos pelos Centros de Especialidades Odontológicas (CEO), enquanto os menores se encontravam no Sul e no Norte ${ }^{20}$.

Além disso, em 2015, os indicadores "Cobertura populacional estimada de equipes de atenção básica" e "Cobertura populacional estimada de equipes básicas de saúde bucal" apontavam que a região Nordeste tinha as maiores taxas de cobertura $(80,25 \%$ e $65,65 \%$, respectivamente), enquanto as regiões Sudeste $(68,15 \%$ e $44,01 \%)$ e Norte $(68,15 \%$ e 47,39$)$ apresentavam as menores ${ }^{15}$.

As barreiras geográficas também podem ser citadas como fatores dificultadores de acesso aos serviços odontológicos. A enorme bacia hidrográfica existente, por exemplo, na região Norte estabelece um hábito cultural da procura espontânea por tratamentos curativos em serviço de referência quaternária e hospitalar, com pequena participação das ações de prevenção de doenças e promoção da saúde $21-22$. Ademais, aspectos como baixa renda, baixa escolaridade e pior percepção da saúde bucal são apresentados como justificativa para menor utilização dos serviços de saúde bucal, bem como dificuldades de organização, estrutura e condições de trabalho desses serviços ${ }^{23-25}$.

Apesar da fraca correlação entre os indicadores "Proporção de exodontia em relação aos procedimentos" e "Média da ação coletiva de escovação dental supervisionada" encontrada por meio da análise espacial bivariada LISA, pode-se inferir uma correlação inversamente proporcional entre eles, fato comprovado pelos dados de 2015 do DataSUS, que mostram menores médias de ação coletiva de escovação dental supervisionada no 


\section{...a inclusão \\ de indicadores \\ socioeconômicos em \\ estudos epidemiológicos \\ que envolvam análise \\ espacial é de extrema importância...}

Norte $(1,03)$ e Nordeste $(1,34)$, enquanto as maiores médias foram encontradas no Centro-0este $(1,98)$, Sudeste $(2,58)$ e Sul $(2,67)^{15}$.

Nesse contexto, regiões com maior dedicação a atividades de promoção da saúde e prevenção de doenças em saúde bucal tendem a apresentar menor proporção de extração dentária em detrimento dos demais procedimentos curativos e preventivos individuais. Entretanto, é perceptível o baixo desenvolvimento de atividades de escovação dental supervisionada nas diversas RIAU do país, fato que pode ser justificado pela falta de investimentos dos gestores no desenvolvimento dessas ações e/ou pela prática eminentemente curativa dos cirurgiõesdentistas.

A enorme extensão do território brasileiro, que agrega grupos populacionais com diferenças econômicas, sociais e culturais entre si, pode explicar as disparidades das condições de saúde bucal encontradas nas 5 grandes regiões do país. Um claro exemplo disso é que as regiões intermediárias de articulação urbana do país com melhores indicadores socioeconômicos, como Índice de desenvolvimento humano e Índice de Gini, tenderam a apresentar valores mais baixos do indicador de proporção de exodontia em comparação aos demais procedimentos de saúde bucal.

A presença de melhor distribuição de renda e de níveis mais elevados de escolaridade está diretamente relacionada a melhor percepção da saúde bucal e, consequentemente, a maior procura dos serviços odontológicos para procedimentos preventivos e curativos em detrimento dos mutiladores ${ }^{23-24}$.

No entanto, apenas a presença de ótimos indicadores de renda, escolaridade e esperança de vida não são suficientes para melhoria significativa das condições de saúde bucal. Tal fato pode ser observado neste estudo: nas RIAU de Fortaleza e Boa Vista, mesmo apresentando elevados valores no Índice de desenvolvimento humano, a má distribuição de renda entre a população ocasionou indicadores de saúde bucal deficientes.

A maior estruturação política e socioeconômica historicamente produzida também é um fator positivo na melhoria das condições de saúde bucal da população, fato que pode ser observado em municípios do Sul e Sudeste que, mesmo apresentando baixos valores no Índice de desenvolvimento humano, tendem a maior utilização dos serviços de saúde bucal - fato que diverge, de modo geral, do Norte e Nordeste, onde, embora pareça haver maior oportunidade de acesso, o impacto das políticas públicas na utilização dos serviços ainda não pôde ser percebido ${ }^{22}$.

Um estudo realizado nas 27 unidades federativas brasileiras apontou uma redução do Índice CPOD (número de dentes cariados, perdidos e obturados) em populações que apresentavam maiores índices de desenvolvimento humano ${ }^{16}$. A partir de dados das 27 capitais brasileiras obtidos pelo levantamento epidemiológico SB Brasil 2010: Pesquisa Nacional de Saúde Bucal - resultados principais, outro estudo agrupou diversos indicadores socioeconômicos (Proporção de pessoas abaixo da linha de pobreza; Proporção de pessoas indigentes; Índice de desenvolvimento humano; Tempo de fluoretação da água de abastecimento; Proporção de domicílios com banheiro; e Proporção de domicílios com água encanada) em um único componente denominado equidade social e encontrou forte relação deste com os desfechos em saúde bucal ${ }^{8}$.

Apesar das limitações intrínsecas dos estudos ecológicos e daqueles que utilizam bancos de dados secundários, a inclusão de indicadores socioeconômicos em estudos epidemiológicos que envolvam análise espacial é de extrema importância, pois fornece subsídios para a tomada de decisões pelos gestores com a finalidade de promover a equidade em saúde entre as populações que experimentam diferentes condições de vida.

\section{CONCLUSÃO}

Diante dos resultados deste estudo, concluise que a cobertura populacional pelos serviços de saúde parece pouco influenciar o indicador referente à mutilação oral da população.

Em contrapartida, atividades de promoção da saúde e prevenção de doenças relativas a saúde bucal e, principalmente, a melhoria dos indicadores socioeconômicos parecem impactar positivamente a 
redução da quantidade de extrações dentárias.

Nesse sentido, pode-se afirmar que a implantação de políticas públicas que visem a reduzir as iniquidades sociais proporcionará melhores condições de saúde bucal à população de todo o Brasil.

\section{CONTRIBUIÇÃO DOS AUTORES}

Adriano de Aguiar Filgueira contribuiu com a realização da pesquisa, o delineamento do estudo e a redação do manuscrito. Angelo Giuseppe Roncalli contribuiu com a redação e a revisão crítica do manuscrito.

\section{REFERÊNCIAS}

1. Silva ET, Oliveira RT, Leles CR. 0 edentulismo no Brasil: epidemiologia, rede assistencial e produção de próteses pelo Sistema Único de Saúde. Tempus (Brasília) [serial on the internet]. 2015 [cited 2018 Nov 5];9(3):121-34. Available from: http://www. tempusactas.unb.br/index.php/tempus/article/ view/1790/1493

2. Brasil. Portaria n. 3.925, de 13 de novembro de 1998. Aprova o Manual para Organização da Atenção Básica no Sistema Único de Saúde. Diário Oficial da União, Brasília (1999 Feb 2); Sec 1.

3. Brasil. Portaria GM/MS n. 21. Aprova a relação dos indicadores da Atenção Básica a serem pactuados entre municípios, estados e Ministério da Saúde. Diário Oficial da União, Brasília (2005 Jan 5); Sec 1.

4. Brasil. Decreto n. 7.508. Regulamenta a Lei n. 8.080, de 19 de setembro de 1990, para dispor sobre a organização do Sistema Único de Saúde SUS, o planejamento da saúde, a assistência à saúde e a articulação interfederativa, e dá outras providências. Diário Oficial da União, Brasília (2011 Jun 28); Sec 1.

5. Brasil. Contrato organizativo da ação pública da saúde. Brasília (DF): Ministério da Saúde; 2011. v. 1.

6. Brasil. Caderno de diretrizes, objetivos, metas e indicadores 2016. Brasília (DF): Ministério da Saúde; 2016.

7. Magri LV, Aciole GG, Salomão FGD, Tagliaferro PS, Ribeiro, LG. Estudo comparativo de indicadores de saúde bucal em município do estado de São Paulo. Saúde Debate [serial on the internet]. 2016 [cited 2018 Nov 5];40(108):144-55. Available from: http:// www.scielo.br/pdf/sdeb/v40n108/0103-1104sdeb-40-108-00144.pdf
8. Bueno RE, Moysés ST, Bueno PAR, Moysés SJ. Determinantes sociais e saúde bucal de adultos nas capitais do Brasil. Rev Panam Salud Pública [serial on the internet]. 2014 [cited 2018 Nov 5];36(1):17-23. Available from: https://scielosp.org/pdf/rpsp/2014. v36n1/17-23/pt

9. Guiotoko SK, Moysés ST, Moysés SJ, França BHS, Bisinelli JC. Iniquidades raciais em saúde bucal no Brasil. Rev Panam Salud Pública [serial on the internet]. 2012 [cited 2018 Nov 5];31(2):2012. Available from: https://www.scielosp.org/article/ ssm/content/raw/?resource ssm path $=/ \mathrm{media} /$ assets/rpsp/v31n2/a07v31n2.pdf

10. Oliveira PMC, Menezes LMB, Santrain MVL, Almeida PC, Almeida MEL. Indicadores de saúde bucal da atenção básica no estado do Ceará: análise crítica. Cadernos ESP [serial on the internet]. 2011 [cited 2018 Nov 5];5(1):29-36. Available from: http://www.esp.ce.gov.br/cadernosesp/index.php/ cadernosesp/article/view/43/40

11. Fernandes LS, Peres MA. Associação entre a atenção básica em saúde bucal e indicadores socioeconômicos municipais. Rev Saúde Pública. $2005 ; 39(6): 930-6$.

12. Palmier AC, Andrade DA, Campos ACV, Abreu MHNG, Ferreira EF. Indicadores socioeconômicos e serviços odontológicos em uma região brasileira desfavorecida. Rev Panam Salud Pública [serial on the internet]. 2012 [cited 2018 Nov 5];32(1):22-9. Available from: https://scielosp.org/pdf/rpsp/2012. v32n1/22-29/pt

13. Fischer TK, Peres KG, Kupek E, Peres MA. Indicadores de atenção básica em saúde bucal: associação com as condições socioeconômicas, provisão de serviços, fluoretação de águas e a Estratégia de Saúde da Família no Sul do Brasil. Rev Bras Epidemiol [serial on the internet] .2010 [cited 2018 Nov 5];13(1):126-38. Available from: http:// www.scielo.br/pdf/rbepid/v13n1/12.pdf

14. Brasil. Projeções populacionais e indicadores socioeconômicos do Brasil e das Unidades de Federação. Rio de Janeiro: Instituto Brasileiro de Geografia e Estatística; 2016.

15. Brasil. Rol de diretrizes, objetivos, metas e indicadores 2013-2015. Brasília (DF): Ministério da Saúde; 2016.

16. Brasil. Caderno de diretrizes, objetivos, metas e indicadores: 2013-2015. 3. ed. Brasília (DF): Ministério da Saúde; 2015. v. 1.

17. Organização das Nações Unidas. Atlas do Desenvolvimento Humano no Brasil. Brasília (DF): Programa das Nações Unidas para o Desenvolvimento; 2016. 
18. Aquilante AG, Aciole, GG. Oral health care after the National Policy on Oral Health - "Smiling Brazil": a case study. Ciênc Saúde Colet [serial on the internet]. 2015 [cited 2018 Nov 5];20(1):23948. Available from: http://www.scielo.br/pdf/csc/ v20n1/pt 1413-8123-csc-20-01-00239.pdf

19. Brasil. SB Brasil 2010: Pesquisa Nacional de Saúde Bucal - resultados principais. Brasília (DF): Ministério da Saúde; 2012.

20. Casotti E, Contarato PC, Fonseca ABM, Borges PK0, Baldani MH. Atenção em saúde bucal no Brasil: uma análise a partir da avaliação externa do PMAQ$A B$. Saúde Debate [serial on the internet]. 2014 [cited 2018 Nov 5];38(Spec):140-57. Available from: http://www.scielo.br/pdf/sdeb/v38nspe/0103-1104sdeb-38-spe-0140.pdf

21. Saliba NA, Moimaz SAS, Fadel CB, Bino LS. Saúde bucal no Brasil: uma nova política de enfrentamento para a realidade nacional. ROBRAC [serial on the internet]. 2010 [cited 2018 Nov 5];18(48):62-6. Available from: http://files.bvs.br/ upload/S/0104-7914/2010/v19n48/a0013.pdf

22. Fernandes JKB, Pinho JR0, Queiroz RCS, Thomaz EBAF. Avaliação dos indicadores de saúde bucal no Brasil: tendência evolutiva pró-equidade? Cad Saúde Pública [serial on the internet]. 2016 [cited 2018 Nov 5];32(2):e00021115. Available from: http:// www.scielo.br/pdf/csp/v32n2/0102-311X-csp-0102311X00021115.pdf

23. Gomes AMM, Thomaz EBAF, Alves MTSSB, Silva AAM, Silva RA. Fatores associados ao uso dos serviços de saúde bucal: estudo de base populacional em municípios do Maranhão, Brasil. Ciênc Saúde Colet [serial on the internet]. 2014 [cited 2018 Nov 5];19(2):629-40. Available from: http://www.scielo. $\mathrm{br} / \mathrm{pdf} / \mathrm{csc} / \mathrm{v} 19 \mathrm{n} 2 / 1413-8123-\mathrm{csc}-19-02-00629 . \mathrm{pdf}$

24. Baldani MH, Antunes JLF. Inequalities in access and utilization of dental services: a cross-sectional study in an area covered by the Family Health Strategy. Cad Saúde Pública [serial on the internet]. 2011 [cited 2018 Nov 5];27(Suppl 2):S272-S283. Available from: http://www.scielo.br/pdf/csp/ $\underline{\mathrm{v} 27 \mathrm{~s} 2 / 14 . \mathrm{pdf}}$

25. Martins P, Aguiar ASW. Acesso aos serviços de saúde bucal na atenção primária à saúde: avanços e desafios da $11^{\text {a }}$ Região de Saúde do Ceará. Sanare (Sobral, Online) [serial on the internet]. 2011;10(1):6-12. Available from: https://sanare. emnuvens.com.br/sanare/article/viewFile/137/129
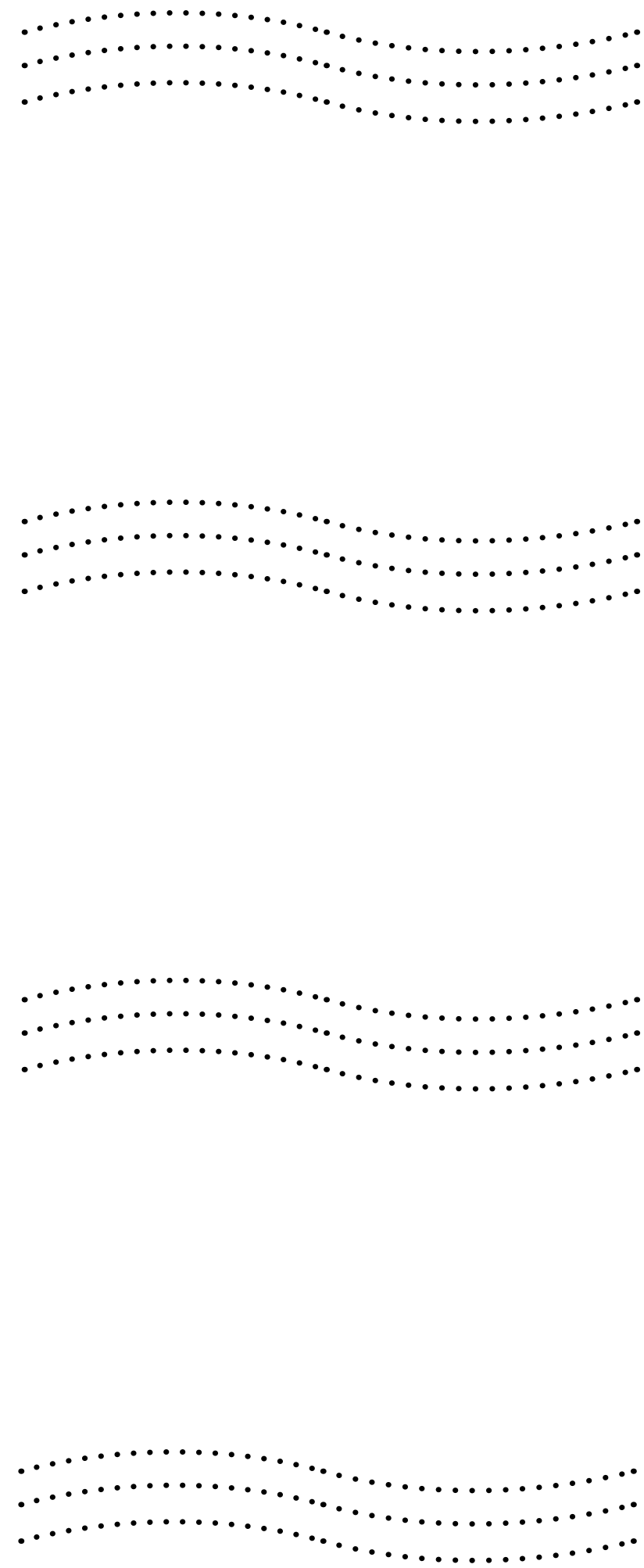

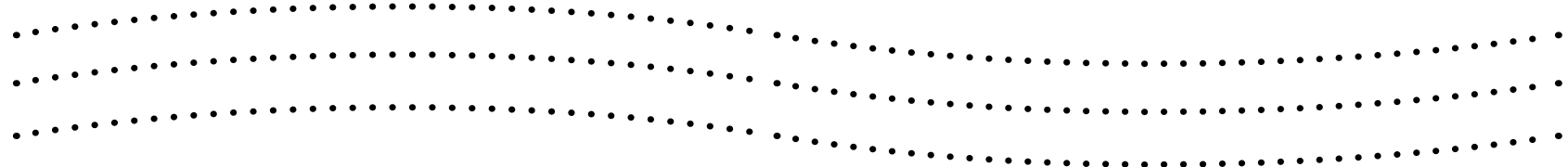

